\title{
Free Energy of Nonequilibrium Polarization Systems. III. Statistical Mechanics of Homogeneous and Electrode Systems*
}

\author{
R. A. Marcus $\dagger$ \\ Department of Chemistry, Polytechnic Institute of Brooklyn, Brooklyn 1, New York
}

(Received 26 April 1963)

\begin{abstract}
A statistical mechanical treatment is given for homogeneous and electrochemical systems having nonequilibrium dielectric polarization. A relation between the free energy of these systems and those of related equilibrium ones is deduced, having first been derived in Part II by a dielectric continuum treatment. The results can be applied to calculating polar contributions in the theory of electron transfers and in that of shifts of electronic spectra in condensed media. The effect of differences in polarizability (of a light emitting or absorbing molecule in its initial and final electronic states) on the polar term in the shift is included by a detailed statistical analysis, thereby extending Part II. Throughout, the "particle" description of the entities contributing to these phenomena is employed, so as to derive the results for rather general potential energy functions.
\end{abstract}

\section{INTRODUCTION}

$\mathbf{P}$ OLAR molecular interactions play a role not only in the usual dielectric properties ${ }^{1}$ but also in a variety of other phenomena, such as solvent effects on the spectra of polar solutes, ${ }^{2}$ homogeneous and electrochemical electron transfers, ${ }^{3}$ intramolecular charge transfers, ${ }^{4}$ and properties of polarons in semiconductors and other materials. ${ }^{5}$ These phenomena usually involve systems with a "nonequilibrium dielectric polarization." 6

In theories of these processes, the calculation of the free energy of the system often plays a central role. In Part $I^{6}$ it was shown by dielectric continuum theory that the polar contribution to the free energy of a nonequilibrium dielectric polarization system equaled the sum of free energies of related equilibrium ones. Consequently, literature calculations of the latter could be immediately applied to the former. Several examples of applications to the theory of electron transfer processes and of spectral shifts were given. However, for noncontinuum discussions a statistical-mechanical derivation of this free energy relation would be desirable. This derivation is given in the present paper. The

\footnotetext{
* Supported in part by the National Science Foundation.

$\dagger$ Alfred P. Sloan Fellow.

I See reviews: W. F. Brown, Handbuch der Physik, edited by S. Flugge (Springer-Verlag, Berlin, 1956), Vol. 17 p. 1; R. H. Cole, Ann. Rev. Phys. Chem. 11, 149 (1960).

2 Y. Ooshika, J. Phys. Soc. Japan 9, 594 (1954); E. Lippert, Z. Naturforsch. 10a, 541 (1955); E. G. McRae, J. Phys. Chem. 61, 562 (1957); R. Platzmann and J. Franck, Z. Physik 138, 411 (1954); R. A. Marcus, paper presented at 133rd National American Chemical Society Meeting, San Francisco (1958); Tech. Rept. No. 4, NSF Grant G-3690 (1958).

${ }_{3}$ R. A. Marcus, J. Chem. Phys. 24, 966 (1956); Can. J. Chem. 37, 155 (1959); Trans. Symp. Electrode Processes Philadelphia, Pa. 1959, 239 (1961); Discussions Faraday Soc. 29, 21 (1960); V. G. Levich and R. R. Dogonadze, Collection Czechoslov. Chem. Communs. 26, 193 (1961) (O. Boshko, translator, University of Ottawa, Ontario), cf. Doki. Akad. Nauk. S.S.S.R. 133, 1368 (1960); N. S. Hush, Trans. Faraday Soc. 57, 557 (1961).

${ }^{4}$ See H. M. McConnell, J. Chem. Phys. 35, 508 (1961).

5 For example, S. I. Pekar, Untersuchungen über die Elektronentheorie der Krystalle (Akademie Verlag, Berlin, 1954); T. Holstein, Ann. Phys. 8, 325, 343 (1959).

6 See, R. A. Marcus, (a) J. Chem. Phys. 24, 979 (1956) (Part I); (b) ibid. 38, 1858 (Part II).
}

analysis also provides some further insight into the continuum description. Applications will be made in subsequent papers.

\section{POTENTIAL-ENERGY FUNCTION}

To minimize the assumptions in the derivation we use the description of a macrosystem given in a recent paper $^{7 \mathrm{a}}$ and applied in Part II: The macrosystem may be considered to be composed of particles, each of which represents a single molecule or a collection of molecules, an entire electrode for example. For some purposes, it suffices to compute functions of the interparticle potential energy. Consequently, detailed assumptions about intraparticle behavior are then unnecessary and can be avoided.

We consider the behavior of the macrosystem at a specified configuration of the nuclei of the "central species," the remainder of the system being called the "medium." Each of these central species $s$ is treated as a separate particle, and the medium is treated as one giant particle $M$. Each central species consists of any molecule of electrode undergoing a transformation in the phenomenon under investigation. If the macrosystem is analyzed at specified positions of the other ions, the central species will be defined to include these ions as well. The subscript $i$ will be used to denote both $s$ and $M$.

Examples of the central species are a pair of reacting molecules, an electrochemically active ion, an electrode, a fluorescing molecule, etc. When one of the central species is an ion this ion plus its inner coordination shell will be regarded as a single particle. When one of the central species is an electrode, the electrode plus

\footnotetext{
7 (a) R. A. Marcus, J. Chem. Phys. 38, 1335 (1963); (b) The polar contribution to $U$ is given by Eq. (19) of this reference. From Eqs. (10), (12), (19), and (34) there, one can show that this polar term depends only on the second powers of the $\rho_{i}{ }^{0}$. For example, if $\rho_{i}{ }^{0}$ is multiplied by a parameter $\nu$ and the corresponding $\Phi_{i}$ are denoted by $\Phi_{i}{ }^{p}$, one can show from these equations that the corresponding $\Phi_{i} \nu / \nu$ satisfy equations independent of $\nu$. That is, $\Phi_{i}{ }^{\nu}$ contains only first powers of the $\nu \rho_{i}{ }^{0}$ and so the polar energy, $\frac{1}{2} \Sigma_{i} \int \nu \rho_{i}{ }^{0} \Phi_{i}{ }^{\nu} d \mathbf{r}$, contains only second powers of $\nu$ and hence only second powers of the $\rho_{i}{ }^{0}$ s (squares and cross products).
} 
any strongly bound adsorbed ions or molecules will also be treated as one particle.

In this paper we are primarily concerned with the nonequilibrium statistically distributed configurations which can arise in a system. We explore in some detail the behavior of the medium outside of these inner coordination shells of the central species. The vibrational motion inside the latter shells can be handled by more standard methods. For this reason, we consider the behavior of the system for any given value of the coordinates in these coordination shells, as well as for any given positional and orientational coordinates of the central species, and for subsequent quantum mechanical treatment, for any given values of the high-frequency coordinates of the medium. Later, one can integrate over these coordinates in accordance with whatever statistical distribution is appropriate, as in Ref. 8. Let $\tau$ denote the coordinates held fixed and $\tau$ denote the other coordinates. We treat the coordinates classically.

For any given $\tau^{f}$ and $\tau$ the electronic energy ${ }^{9} U_{\text {tot }}$ is the sum of an intraparticle term and of an interparticle one. The former is the total electronic energy when the particles are isolated from each other and have the intraparticle configuration contained in this value of $\left(\tau^{f}, \tau\right)$. The second term is the change on bringing the particles together, to the interparticle configuration specified by this $\left(\tau^{f}, \tau\right)$.

We may therefore write $U_{\text {tot }}$ as

$$
U_{\text {tot }}=U+U^{f},
$$

where $U$ is the sum of the intraparticle term for the medium and of all interparticle terms. $U^{f}$ is the intraparticle term for the central species and is a function of $\tau^{f}$ alone.

The interparticle electronic energy itself will be taken to be the sum of polar and "nonpolar" or, more precisely, "electron correlation" terms, the latter including repulsion and London dispersion energies of interactions between the particles. ${ }^{\mathrm{a}}$ We then expand the interparticle energy in terms of the $\rho_{i}{ }^{\prime}$ 's, the charge densities on the isolated particles at the intraparticle configuration given by $(\tau, \tau)$, retaining terms up to second powers in the $\rho_{i}{ }^{9}$ 's. ${ }^{9 \mathrm{a}}$ These terms will contain only zero and second powers of the $\rho_{i}^{0}$ 's. ${ }^{7}$ They may be conveniently classified further according as they contain $z$ zero, first, or second powers of the $\rho_{i}{ }^{\text {' }}$ 's of the central species, $\rho_{s}{ }^{0}$, namely as $U(0), U(1)$, and $U(2)$, respectively:

$$
U=U(0)+U(1)+U(2) \text {. }
$$

\footnotetext{
${ }^{8}$ R. A. Marcus, J. Chem. Phys. (to be published).

9 This electronic energy serves, of course, as a potential-energy function for nuclear motion, in the adiabatic approximation, in the standard way.

${ }_{93}$ Note added in proof: This type of expansion, with retention of second powers of the permanent charge densities, neglects "electronic dielectric saturation" and is common to practically all existing calculations in the literature. The latter employ additional assumptions as well, and so represent special cases of $\mathbf{E q}$.

(1) [cf. Ref, 7(a)].
}

$U(2)$ must be independent of $\rho_{M^{0}}, U(1)$ must contain first powers of $\rho_{M}{ }^{0}$ and hence vanish when either $\rho_{M}{ }^{0}$ or $\rho_{s}{ }^{0}$ vanishes, and $U(0)$ contains both zero and second powers of $\rho_{M^{0}}$. The part of $U(0)$ which has zero powers of $\rho_{M}{ }^{0}$ consists of the intraparticle term for $M$ and of the interparticle nonpolar term. Each term in (2) depends not only on $\tau$ but also on $\tau^{f}$.

\section{STATISTICAL MECHANICS OF EQUILIBRIUM AND NONEQUILIBRIUM POLARIZATION SYSTEMS}

In the systems considered here the configurational distribution of the "medium" may or may not be the equilibrium one for the specified electronic state of the central species. As in Parts I and II, these systems are called equilibrium and nonequilibrium polarization systems, respectively.

For a medium having an equilibrium configurational distribution, the configurational contribution of the $\tau$ coordinates to the free energy of the system at the specified $\tau^{f}$ is:

$$
F_{c}=-k T \ln \int \exp \left(\frac{-U}{k T}\right) d \tau
$$

where, for brevity, we have omitted the usual product of factorials that takes care of indistinguishability of like molecules. (This product cancels in all of the freeenergy differences in this paper.) The relation of $F_{c}$ to the total configurational contribution to the free energy is noted in Appendix I.

The polar contribution to $F_{c}$ is defined as $F_{c}$ minus its value $F_{c}(0)$ when all $\rho_{8}{ }^{0}$ vanish. Denoting this polar term by $F$ we thus obtain from (2) and (3):

$$
\begin{aligned}
F=- & k T \\
& \times \ln \left(\int \exp \left(\frac{-U}{k T}\right) d \tau / \int \exp \left[\frac{-U(0)}{k T}\right] d \tau\right) .
\end{aligned}
$$

We also require the configurational free energy of a system in which the medium responds to the $\rho_{s}{ }^{0}$ of the central species via an electronic polarizability rather than via an adjustment of its nuclear configurational distribution. The distribution function for the coordinates in this "equilibrium optical polarization system" is the same as the one in which the $\rho_{s}{ }^{0}$ vanish, and so is proportional to $\exp [-U(0) / k T]$. The polar contribution $F^{\text {op }}$ to the free energy of this system is the polar energy of interaction of the central species with each other and with the medium, $U(1)+U(2)$, averaged with respect to this distribution

$$
\begin{aligned}
F^{\mathrm{op}}=\int & {[U(1)+U(2)] } \\
& \times \exp \left[\frac{-U(0)}{k T}\right] d \tau / \int \exp \left[\frac{-U(0)}{k T}\right] d \tau .
\end{aligned}
$$

Considering nonequilibrium polarization systems next, those that we have investigated thus far have a configurational distribution function appropriate to 
some charge distribution on the central species, but not to the existing one. However, the electronic polarization of the system, which has a very short relaxation time, is that which is appropriate to the existing $\rho_{s}{ }^{0}$ 's and to the existing orientation-atomic polarization of the medium. We use a subscript 0 to denote the properties of the state for which the configurational distribution function of the noncentral species would be the equilibrium one at the specified configuration of the central species. This state was called the "equivalent equilibrium system" (e.e.s.). ${ }^{10}$

An example of a nonequilibrium polarization system is one in which a central species has just absorbed or emitted light: the configurational distribution of the surrounding molecules is appropriate to the molecular state just before but not after the transition. Again, in the activated complex of electron transfer processes, the configurational distribution of molecules near the reacting pair (or near a reacting ion and electrode) is appropriate to some hypothetical charge distribution, a compromise between that of the reactants and that of the products, but not to the existing one..$^{10}$

The $\tau$ contribution to the free-energy difference $F_{c}{ }^{\text {non }}-F_{c_{0}}$ between the nonequilibrium state and its e.e.s. equals the energy difference, since both have the same configurational distribution and, thereby, the same entropy:

$$
\begin{aligned}
F_{c}^{\text {non }}-F_{c_{0}} & =\int\left(U_{1}-U_{0}\right) \\
& \times \exp \left(\frac{-U_{0}}{k T}\right) d \tau / \int \exp \left(\frac{-U_{0}}{k T}\right) d \tau,
\end{aligned}
$$

where $U_{1}$ and $U_{0}$ denote the potential energy $U$ when the $\rho_{i}{ }^{0}$ 's are those in the nonequilibrium polarization system, $\rho_{i_{1}}{ }^{0}$, say, and those in the e.e.s., $\rho_{i_{0}}{ }^{0}$, respectively (we note that $\rho_{M_{1}}{ }^{0}=\rho_{M_{0}}{ }^{0}$ ).

The relation of this $\tau$ contribution to the total configurational free energy difference of nonequilibrium and equivalent equilibrium systems is described in Appendix I.

Because of the assumed additivity of the interparticle polar and nonpolar terms, $U_{1}-U_{0}$ in (6) can be written as the sum of two differences, one due to the difference in polar properties of the central species, $U_{1}(1)+U_{1}(2)-U_{0}(1)-U_{6}(2)$, the other due to the difference in their nonpolar properties, $U_{1}(0)-U_{0}(0)$. We may define the polar contribution to (6) to be given by $(7)$, write it as $F^{\text {non }}-F_{0}$, and thereby define $F^{\text {non }}$.

$$
F^{\mathrm{non}}-F_{0}=\left\langle U_{1}(1)+U_{1}(2)-U_{0}(1)-U_{0}(2)\right\rangle_{0},
$$

where

$$
\langle f\rangle_{0}=\int f \exp \left(\frac{-U_{0}}{k T}\right) d \tau / \int \exp \left(\frac{-U_{0}}{k T}\right) d \tau .
$$

In the polar term there occurs a generalized polariza${ }^{10}$ R. A. Marcus, Discussions Faraday Soc. 29, 21 (1960). bility operator $A_{i}$ of the central species. ${ }^{7 \mathrm{a}} A_{i}$ may differ in the non and [0] systems. For example, if the nonequilibrium state is formed from an equilibrium one [0] by light emission, the polarizability of the fluorescing molecule differs in the initial and final electronic states. The rhs of Eq. (7) can be rewritten as

$$
\left\langle U\left(\rho_{i_{1}}{ }^{0}, A_{i_{1}}\right)-U\left(\rho_{i_{0}}{ }^{0}, A_{i_{0}}\right)\right\rangle_{\nu_{0}} .
$$

Accordingly, (7) can be re-expressed as the sum of two terms, one at fixed $\rho_{i}{ }^{0}$ and the other at fixed $A_{i}$ :

$$
\begin{aligned}
F^{\mathrm{non}}-F_{0}=\left\langle U\left(\rho_{i_{1}}{ }^{0}, A_{i_{1}}\right)-U\left(\rho_{i_{1}}{ }^{0}, A_{i_{0}}\right)\right\rangle_{0} \\
+\left\langle U\left(\rho_{i_{1}}{ }^{0}, A_{i_{0}}\right)-U\left(\rho_{i_{0}}{ }^{0}, A_{i_{0}}\right)\right\rangle_{0} .
\end{aligned}
$$

The first difference on the rhs is calculated in one of the concluding sections of this paper and is given by Eq. (35). The second difference in (9) is the same as the mean polar energy difference when the corresponding $A_{i}$ 's in the nonequilibrium and equivalent equilibrium systems are identical. It is computed first for the case of a dielectrically unsaturated system. Equation (14) is thereby derived. Equation (18) is then derived for partially dielectrically saturated systems under an approximation milder than the one of dielectric unsaturation.

In summary, the value of $F^{\text {non }}-F_{0}$ will be given by the sum of the rhs of Eqs. (14) or (18) and (35). For the usual electron transfer reactions the contribution of (35) may be ignored. Only if there is a large difference in polarizabilities in the initial and final states, as in some spectral shifts, need it be considered. In the following calculations we need to refer to four systems in which the configurational distribution function is the equilibrium one at the specified $\tau^{f}$ and at the cited $\rho_{s}^{0}$ 's:

(i) System [0], the e.e.s. just described, $\rho_{s}{ }^{0}=\rho_{s 0}{ }^{0}$,

(ii) System [1], $\rho_{s}{ }^{0}=\rho_{s_{1}}{ }^{0}$,

(iii) System $[1-0], \rho_{s}^{0}=\rho_{s_{1}-0}{ }^{0}=\rho_{s_{1}}{ }^{0}-\rho_{s_{0}}{ }^{0}$,

(iv) System $[1-0, o p], \rho_{8}{ }^{0}=\rho_{8_{1}}{ }^{0}-\rho_{8_{0}}{ }^{0}$ and the medium responds to these $\rho_{s}^{0}$ 's only via its electronic polarization.

If in the "non" system the interparticle nonpolar forces are the same as those in the $[0]$ system, $U_{1}(0)$ and $U_{0}(0)$ are equal, for the only other term in $U(0)$ is the same in both systems, being an intraparticle term for $M$. In many electron-transfer systems of interest the central species consist of an electrode and/or ions plus inner coordination shells of configuration $\tau^{j}$. In this case, we may take as a good approximation $U_{1}(0)=$ $U_{0}(0)$ and $A_{i_{0}}=A_{i_{1}}$.

On the other hand, if the interparticle nonpolar forces and $A_{\text {s }}$ differ in the two systems, then the $F_{1}$ which appears in the following discussion refers to a hypothetical system [1] having the same medium, the same $A_{s}^{\prime}$ 's and the same $U(0)$ as the [0] system, but having $\rho_{\varepsilon}^{0}=\rho_{t 1}{ }^{0}$. 


\section{DIELECTRICALLY UNSATURATED SYSTEMS}

Dielectric unsaturation is characterized by a linear response of the medium to the $\rho_{i}{ }^{0}$ of the central species. If the coordinates of any ions in the medium are included in $\tau$, then the introduction of a linear response approximation will give rise not only to dielectric unsaturation but also to a Debye-Hückel approximation for salt effects as well. To avoid the second of these we hold the ionic coordinates fixed by including them among the $\tau^{f}$ (i.e., every ion is regarded as a central species). This constraint need not be imposed in the milder approximation of partial dielectric unsaturation.

Because of the linear response of the medium to the $\rho_{s}{ }^{0}$ 's the polar contribution to the free energy at given $\tau^{f}$ is quadratic in the $\rho_{s}{ }^{\prime}$ 's. Accordingly, this approximation may be introduced by multiplying each $\rho_{s}{ }^{0}$ by a parameter $\lambda$, then expanding the free energy in a Taylor's series in $\lambda$ about $\lambda=0$ retaining terms up to $\lambda^{2}$, and finally setting $\lambda=1$. [In (2), the $U(1)$ and $U(2)$ are therefore multiplied by $\lambda$ and $\lambda^{2}$, respectively. $]$ In this way, Eqs. (4) and (5) yield

$F=\langle U(1)\rangle+\langle U(2)\rangle-(1 / 2 k T)\left[\left\langle U(1)^{2}\right\rangle-\langle U(1)\rangle^{2}\right]$

where

$$
F^{\circ} \mathrm{p}=\langle U(1)\rangle+\langle U(2)\rangle
$$

$$
\langle f\rangle=\int f \exp \left[\frac{-U(0)}{k T}\right] d \tau / \int \exp \left[\frac{-U(0)}{k T}\right] d \tau .
$$

In the same way we also obtain (13) from (7).

$$
\begin{aligned}
F^{\mathrm{non}}-F_{0}= & \left\langle U_{1}(1)-U_{0}(1)+U_{1}(2)-U_{0}(2)\right\rangle \\
+ & (1 / 2 k T)\left\langle U_{1}(1)-U_{0}(1)\right\rangle\left\langle U_{0}(1)\right\rangle \\
& -(1 / 2 k T)\left\langle\left[U_{1}(1)-U_{0}(1)\right] U_{0}(1)\right\rangle,
\end{aligned}
$$

where \langle\rangle is as defined in (12) with $U(0)$ given by $U_{0}(0)$.

We next introduce the two hypothetical dielectrically unsaturated systems mentioned earlier, $[1-0],[1-0$, op] whose polar contributions to the free energy are given by (10) and (11) with appropriate subscripts; we define $U_{1-0}(0)$ as $U_{0}(0)$ and $A_{s_{1-0}}$ as $A_{s_{0}}$. Combining these two equations with a corresponding equation for $F_{1}$ we obtain (14).

$$
F^{\text {non }}-F_{1}=F_{1-0}{ }^{\text {op }}-F_{1-0} .
$$

This equation has been applied to discussions of electron transfer processes. ${ }^{6 \mathrm{~b}, 8}$ For spectral shifts, one needs instead $F^{\text {non }}-F_{0}$.

$$
F^{\mathrm{non}}-F_{0}=F_{1}-F_{0}+F_{1-0}^{\mathrm{op}}-F_{1-0} .
$$

\section{PARTIAL DIELECTRIC SATURATION}

Partial dielectric saturation associated with the coordinates $\tau$ can occur outside the inner coordination shell of any highly charged ion. The inner coordination shell itself is largely "saturated," but it depends on $\tau^{f}$ and so is unaffected by any unsaturation approximation. When partial $\tau$-coordinate saturation occurs, the expansions of the previous section are inadequate. However, when the change in charge of each particle on going from system [0] to [1] is small, as it typically appears to be, we may expand $F_{1}$ about $F_{0}$ in powers of the differences $\left(\rho_{s_{1}}{ }^{0}-\rho_{s_{0}}{ }^{0}\right)$ 's. We retain terms up to the second power in these differences. This expansion may be performed by introducing a parameter $\lambda$ such that $\rho_{s}{ }^{0}$ is replaced by $\rho_{s 0}{ }^{0}+\lambda\left(\rho_{s_{1}}{ }^{0}-\rho_{s_{0}}{ }^{0}\right)$ wherever $\rho_{\varepsilon_{1}}{ }^{0}$ appears. One then expands $F_{1}$ in a Taylor's series in $\lambda$ about $\lambda=0$, retaining terms up to $\lambda^{2}$ and finally setting $\lambda=1$. We thereby obtain (16). Since we no longer assume a linear response to the $\rho_{s_{0}}{ }^{3}$ 's, it may often be useful to regard all ions except those undergoing the transformation to be noncentral species, i.e., to have coordinates which are not held fixed and so belong to the totality $\tau^{8}$ Equation (16) applies regardless of which of the two alternative ways for regarding these atmospheric ions is adopted.

$$
\begin{aligned}
& F_{1}-F_{0}=\left\langle U_{1}(1)-U_{0}(1)\right\rangle_{0}+\left\langle U_{1}(2)-U_{0}(2)\right\rangle_{0} \\
& -(1 / 2 k T)\left\{\left\langle\left[U_{1}(1)-U_{0}(1)\right]^{2}\right\rangle_{c}-\left\langle U_{1}(1)-U_{0}(1)\right\rangle_{0}^{2}\right\},
\end{aligned}
$$

where

$$
\langle f\rangle_{0}=\frac{\int f \exp \left(\frac{-U_{0}(0)+U_{0}(1)+U_{0}(2)}{k T}\right) d \tau}{\int \exp \left(\frac{-U_{0}(0)+U_{0}(1)+U_{0}(2)}{k T}\right) d \tau} .
$$

We again introduce two dielectrically unsaturated systems $[1-0]^{\prime}$ and $[1-0 \text {,op }]^{\prime}$, where $U_{1-0}(0)$ is now defined as numerically equal to $U_{0}(0)+U_{0}(1)+U_{0}(2)$ for every value of $\tau .^{11}$ The dielectric unsaturation approximation is introduced by multiplying the $\rho_{s_{1}-0}{ }^{0} \mathrm{~s}$ in $U_{1-0}(1)$ and $U_{1-0}(2)$ by $\lambda$ and then proceeding as in the previous section. From the resulting equations and from (13) and (16) we then obtain (18):

$$
F^{\mathrm{non}}=F_{1}+F_{1-0^{\mathrm{op}}}-F_{1-0^{\prime}} .
$$

Equations (14) and (18) were derived in Part II using the dielectric continuum approximation ${ }^{6 \mathrm{~b}}$ Several applications of (14) were made there, and application of (18) may be found in Ref. 8 .

In view of the fact that Eqs. (14) [and (18)] have derived both on the basis of statistical mechanical and continuum methods, it is of interest to compare two approaches in their treatment of nonpolar and optical polarization media, and to consider the possibility of

${ }^{11}$ Within the approximation employed, one can show that (18) would again be obtained if $U_{1-0}(0)$ were defined as $U_{1}(0)+$ $U_{1}(1)+U_{1}(2)$, or as any function intermediate between this one and $U_{0}(0)+U_{0}(1)+U_{0}(2)$ : We note from (10) and (11) that

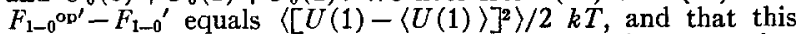
average of a fluctuation term is, within the partial unsaturation approximation, the same regardless of which of the above values of $U_{1-0}(0)$ is used in computing the average with Eq. (12). 
deriving (14) or (18) for the case where "op" denotes a nonpolar medium rather than an optical polarization one.

\section{COMPARISON OF NONPOLAR AND OPTICAL POLARIZATION MEDIA}

A nonpolar medium is defined as one for which $\rho_{M}{ }^{0}$ equals zero for all $\tau$. For such a medium, the polar term in $U$ reduces to $U(2)$ and Eq. (10) for $F$ becomes:

$$
F=\langle U(2)\rangle^{*}
$$

where \langle\rangle$^{*}$ denotes an average with respect to

$$
\exp \left[\frac{-U^{*}(0)}{k T}\right] / \int \exp \left[\frac{-U^{*}(0)}{k T}\right] d \tau,
$$

the ${ }^{*}$ indicating that in $U^{*}(0) \rho_{M}{ }^{0}$ vanishes. Equation (19) may be compared with the $F$ of an optical polarization system, given by $(20)$.

$$
F^{\mathrm{op}}=\langle U(1)+U(2)\rangle
$$

These two F's are equal when: (i) the medium is treated as a dielectric continuum. \{This treatment can be regarded as a limiting case of the statistical mechanical one in which the ratio of size of the central species to that of each molecule composing the medium becomes extremely large. In this case, the nonpolar forces between the central species and the molecules of the medium do not orient the latter molecules preferentially, and $\langle U(1)\rangle$ in Eq. (20) then vanishes. If one chooses $U^{\text {op }}(0)=U^{*}(0)$ for all $\tau$, expressions (19) and (20) are then equal. In this instance, the [op] system in (14) or (18) could also be interpreted as one having a nonpolar equilibrium polarization medium.\} or (ii) the molecules of the medium have an appropriate symmetry property, namely one where after a change of sign of the charge density on the molecule, a subsequent suitable rotation restores the molecule to its original geometry, its original $\rho_{i}{ }^{0}$ and its original $A_{i}$ : Application of this symmetry operation shows that to each configuration $\tau$ there is one of equal probability having the same magnitude of $U(1)$ but opposite in sign. $\langle U(1)\rangle$ in Eq. (20) then vanishes, and the choice $U^{*}(0)=U^{\mathrm{op}}(0)$ for all $\tau$ then makes (19) and (20) equal. Once again, the op in (14) or (18) can be then interpreted as "nonpolar medium." Any actual molecule would satisfy such a symmetry requirement in an approximate way at most.

The foregoing arguments provide some further insight into one aspect of the continuum and statistical mechanical derivations of (14) [and (18)]. This equation was first derived in Part II using continuum theory. However, the usual dielectric continuum method does not distinguish between nonpolar and "optical polarization" systems. The properties of both are characterized only by an optical dielectric constant. In the statistical mechanical treatment, on the other hand, we saw that there is in general a difference in these two systems, and that Eq. (14) applied in that case only when op denotes an optical polarization system. When this statistical treatment was converted into a continuum one, as described in Condition (i) or when Conditions (ii) prevailed, "op" could denote a nonpolar system also.

\section{"CHANGE OF POLARIZABILITY" CONTRIBUTION TO EQ. (9)}

We compute the first term in the rhs of (9), to firstorder differences in $A_{s_{1}}-A_{s_{0}}$. We recall from a recent particle description of the system ${ }^{7 a}$ that the polar contribution to $U$ can be written as

$$
U^{\mathrm{pol}}=\frac{1}{2} \sum_{i} \int \Phi_{i}(\mathbf{r}) \rho_{i}{ }^{0}(\mathbf{r}) d \mathbf{r},
$$

where $\Phi_{i}(\mathbf{r})$ is the potential at any point $\mathbf{r}$ in the system, minus the self-potential of particle $i$. Equation (22) represents a set of equations which may be solved for the $\Phi_{i}$ 's.

$$
\Phi_{i}\left(r^{\prime}\right)=\sum_{j \neq i} \int\left[\rho_{j}{ }^{0}(\mathbf{r})+A_{j}\left(\mathbf{r}, \mathbf{r}^{\prime \prime}\right) \Phi_{j}\left(\mathbf{r}^{\prime \prime}\right) \frac{d \mathbf{r}}{r}\right] .
$$

The nonpolar contribution to $U$ cancels in (9), it will be recalled, because of the earlier decomposition of the total change of $U$ made in obtaining (2).

To perform the calculation we use the device employed earlier of introducing a parameter $(\gamma$ now $)$ and differentiating with respect to it. Let

$$
A_{i}{ }^{\gamma}=A_{i_{0}}+\gamma\left(A_{i_{1}}-A_{i_{0}}\right),
$$

where $\gamma$ varies from 0 to 1 . The desired first term in the rhs of (9) becomes:

$$
\left\langle U\left(\rho_{i_{1}}{ }^{0}, A_{i_{1}}\right)-U\left(\rho_{i_{1}}{ }^{0}, A_{i_{0}}\right)\right\rangle_{0}=\left\langle U^{\gamma=1}-U^{\gamma=0}\right\rangle_{0},
$$

where \langle\rangle$_{0}$ is defined by (17), and in $U^{\gamma=1}, U^{\gamma=0}$ one has $\rho_{s}{ }^{0}=\rho_{s_{1}}{ }^{0}$. Expanding $U^{\gamma}$ in a Taylor's series about $\gamma=0$, we have:

$$
U^{\gamma}=U^{\gamma=0}+\left(\frac{\partial U^{\gamma}}{\partial \gamma}\right)_{\gamma=0} \gamma+\left(\frac{\partial^{2} U^{\gamma}}{\partial \gamma^{2}}\right)_{\gamma=0} \frac{\gamma^{2}}{2 !}+\cdots
$$

Differentiating (21) and (22) with respect to $\gamma$ after introducing (23) and (25), we find

$$
\begin{gathered}
\frac{\partial U^{\gamma}}{\partial \gamma}=\frac{1}{2} \sum_{i} \int \frac{\partial \Phi_{i}{ }^{\gamma}}{\partial \gamma} \rho_{i}{ }^{0} d \mathbf{r} \\
\frac{\partial \Phi_{i}^{\gamma}}{\partial \gamma}=\sum_{j \neq i} \int\left(\frac{\partial A_{j}^{\gamma}}{\partial \gamma} \Phi_{j}^{\gamma}+A_{j}{ }^{\gamma} \frac{\partial \Phi_{j}^{\gamma}}{\partial \gamma}\right) \frac{d \mathbf{r}}{r} .
\end{gathered}
$$

Comparing (26) with (22), $\partial \Phi_{i}{ }^{\gamma} / \partial \gamma$ is seen to be the same as the potentials $\Phi_{i}^{+}$of a system $[+]$in which $A_{j}{ }^{+}=A_{j}^{\gamma}$ and $\rho_{j}{ }^{++}=\left(\partial A_{j}^{\gamma} / \partial \gamma\right) \Phi_{j}^{\gamma}$. We may convert (26) to a more useful form using an equation proved elsewhere $^{7 \mathrm{a}}$ :

$$
\sum_{i} \int \Phi_{i}{ }^{a} \rho_{i}{ }^{0 b} d \mathbf{r}=\sum_{i} \int \Phi_{i}{ }^{b} \beta_{i}{ }^{0 a} d \mathbf{r}
$$


where $[a]$ and $[b]$ are any two systems such that $A_{i}{ }^{a}=A_{i}{ }^{b}$. We let $a$ denote the [+] system and $b$ the system described by $\gamma$ (both have $A_{i}=A_{i}^{\gamma}$.) Equations (26) and (28) then yield (29), on noting that $\partial A_{M^{\gamma}} / \partial \gamma$ equals zero.

$$
\frac{\partial U \gamma}{\partial \gamma}=\frac{1}{2} \sum_{s} \int \Phi_{s} \frac{\partial A_{s}{ }^{\gamma}}{\partial \gamma} \Phi_{s}^{\gamma} d \mathbf{r} .
$$

Equation (29) is general for any system, regardless of the value of $\rho_{s}{ }^{0}$ or of whether the system is an equilibrium or a nonequilibrium polarization system. We need to evaluate $\left\langle\partial U^{\gamma} / \partial \gamma\right\rangle_{0}$ to compute (24):

$$
\left\langle\frac{\partial U \gamma}{\partial \gamma}\right\rangle_{0}=\frac{1}{2}\left\langle\sum_{s} \int \Phi_{s}{ }^{\gamma} \frac{\partial A_{s}^{\gamma}}{\partial \gamma} \Phi_{s}^{\gamma} d \mathbf{r}\right\rangle_{0}
$$

To evaluate (30), we proceed indirectly. If $F^{\gamma}$ is the polar contribution to an equilibrium polarization system whose $A_{i}$ is $A_{i}{ }^{\gamma}$, differentiation of (4) leads to

$$
\frac{\partial F^{\gamma}}{\partial \gamma}=\int\left(\frac{\partial U \gamma}{\partial \gamma}\right) \exp \left(\frac{-U^{\gamma}}{k T}\right) d \tau / \int \exp \left(\frac{-U^{\gamma}}{k T}\right) d \tau \text {. }
$$

Hence, from (29) and (31) we have

$$
\begin{aligned}
\frac{\partial F^{\gamma}}{\partial \gamma}=\int\left(\frac{1}{2} \sum_{s} \int \Phi_{s} \gamma \frac{\partial A_{s}{ }^{\gamma}}{\partial \gamma} \Phi_{s}^{\gamma} d \mathbf{r}\right) \\
\quad \times \exp \left(\frac{-U^{\gamma}}{k T}\right) d \tau / \int \exp \left(\frac{-U^{\gamma}}{k T}\right) d \tau .
\end{aligned}
$$

The evaluation of (30) would therefore be immediate if the averaging in (30) had been with respect to $\exp \left(-U^{\gamma} / k T\right)$ instead of with respect to $\exp \left(-U_{0} / k T\right)$. Instead, we rewrite to Eq. (30) identically as

$$
\begin{aligned}
\left\langle\frac{\partial U^{\gamma}}{\partial \gamma}\right\rangle_{0}=\frac{1}{2} \sum_{s} & \left\langle\int\left(\Phi_{s} \gamma-\Phi_{s_{0}}\right) \frac{\partial A_{s}^{\gamma}}{\partial \gamma}\left(\Phi_{s}^{\gamma}-\Phi_{s_{0}}\right) d \mathbf{r}\right\rangle_{0} \\
& +\frac{1}{2} \sum_{s}\left\langle\int \Phi_{s_{0}} \frac{\partial A_{s}^{\gamma}}{\partial \gamma} \Phi_{s_{0}} d \mathbf{r}\right\rangle_{0} \\
& +\sum_{s}\left\langle\int\left(\Phi_{s}^{\gamma}-\Phi_{s_{0}}\right) \frac{\partial A_{s}^{\gamma}}{\partial \gamma} \Phi_{s_{0}} d \mathbf{r}\right\rangle_{0}
\end{aligned}
$$

where we have used the Hermitian property ${ }^{7 a}$ of $\partial A^{\gamma} / \partial \gamma$, inherited from $A_{s}{ }^{\gamma}$, and where 0 denotes, as before, a system having $\rho_{s}{ }^{0}=p_{s_{0}}{ }^{0}$ and $A_{s}=A_{s_{0}}$.

At $\gamma=0, \Phi_{s}{ }^{\gamma}$ refers to a system having the same $\rho_{s}$ and $A_{6}$ as the [1] system, or more precisely as the hypothetical [1] system defined earlier, namely $\rho_{s_{1}}$ and $A_{s_{0}}$. Its other properties are denoted by a subscript 1 , so $\Phi_{8}{ }^{\gamma}$ at $\gamma=0$ equals $\Phi_{8_{1}}$. The $\Phi_{8}$ of system [0] is $\Phi_{8_{0}}$. Subtracting Eq. (22) for $\Phi_{\varepsilon_{0}}$ from that for $\Phi_{8_{1}}$ one can see that $\Phi_{s_{1}}-\Phi_{s_{0}}$ is the $\Phi_{\varepsilon}(\mathbf{r})$ of a system having a nonpolar medium and having $\rho_{s}{ }^{0}=\rho_{s 1}{ }^{0}-\rho_{s 0}{ }^{0}$. We denote its properties by $1-0$ and $n p$ subscripts or superscripts. For example, $\Phi_{s_{1}}-\Phi_{s_{0}}$ equals $\Phi_{s_{1}-0}{ }^{n p}$. The usual rotational and translational flunctuations in a condensed nonpolar medium have relatively little effect on its $\Phi_{i}$, so $\Phi_{s_{1}}-\Phi_{\varepsilon_{0}}$ can be replaced by its average value. Again, since the coordinates of $s$ are held fixed when \langle\rangle$_{0}$ is computed, $\partial A_{s} \gamma / \partial \gamma$ and \langle\rangle$_{0}$ commute. At $\gamma=0$, Eq. (33) now becomes (34), since $\Phi_{s_{1-0}}^{n p}$ is insensitive to the polar properties of the averaging function.

$$
\begin{aligned}
\left\langle\left(\frac{\partial U \gamma}{\partial \gamma}\right)_{\gamma=0}\right\rangle_{0} & =\frac{1}{2} \sum_{s}\left\langle\int \Phi_{s_{1}-0}{ }^{n p} \frac{\partial A_{s}^{\gamma}}{\partial \gamma} \Phi_{s_{1}-0}{ }^{n p} d \mathbf{r}\right\rangle_{1-0, n p} \\
& +\frac{1}{2} \sum_{s}\left\langle\int \Phi_{s_{0}} \frac{\partial A_{s}^{\gamma}}{\partial \gamma} \Phi_{s_{0}} d \mathbf{r}\right\rangle_{n} \\
& +\sum_{s} \int\left\langle\Phi_{s_{1}-0}{ }^{n p}\right\rangle_{1-0, n p} \frac{\partial A_{s}{ }^{\gamma}}{\partial \gamma}\left\langle\Phi_{s}\right\rangle_{0} d \mathbf{r}
\end{aligned}
$$

Using (32) for both the $[0]$ and $[1-0, n p]$ equilibrium polarization systems, we note that the first term on the rhs of (34) equals $\left(\partial F_{1-0, n p} \gamma / \partial \gamma\right)_{\gamma=0}$ and the second equals $\left(\partial F_{0} \gamma / \partial \gamma\right)_{\gamma=0}$. The third involves a typical average and we give an example of its calculation later.

We have not yet introduced the approximation of evaluating $\left\langle U^{\gamma=1}-U^{\gamma=0}\right\rangle$ to first-order differences in $A_{s_{1}}-A_{s_{0}}$. To do so it suffices to neglect powers of $\gamma^{2}$ and higher in the expansion of (24) and then to set $\gamma=1$. Calling $U^{\gamma=1}-U^{\gamma=0}, \Delta U$ and calling $A_{s_{1}}-A_{s_{0}}$, $\Delta A_{s}$ we finally obtain (35).

$$
\begin{aligned}
\langle\Delta U\rangle_{0}=\left(\frac{\partial F_{1-0^{n p \gamma}}}{\partial \gamma}\right)_{\gamma=0}+\left(\frac{\partial F_{0}^{\gamma}}{\partial \gamma}\right)_{\gamma=0} & \\
& +\sum_{s} \int\left\langle\Phi_{s_{1}-0}{ }^{n p}\right\rangle_{\mathbf{l}-0, n p} \Delta A_{s}\left\langle\Phi_{s_{0}}\right\rangle_{v} d \mathbf{r} .
\end{aligned}
$$

In summary, we recall that the polar contribution $F^{\text {non }}-F_{0}$ in Eq. (9) is given by the sum of the rhs of Eqs. (14) $[$ or (18)] and (35).

In the next section we apply (35) to a common model used in the literature, Eq. (14) having been applied to the same model in Part II.

\section{APPLICATION OF EQ. (35) TO A DIPOLAR SOLUTE IN A CONTINUUM}

We consider the model of a single dipolar solute molecule $s$, treated as a sphere of radius $a$, possessing a dipole of moment $\boldsymbol{u}$ at its center, a polarizability $\alpha$, and imbedded in the solvent treated as a dielectric continuum. Equation (35) can readily be applied to more complex models, however. Since we employ a dielectric continuum model, the nonpolar and optical polarization systems are equivalent, and we can replace $n p$ in Eq. (35) by op.

If $\mathbf{r}_{s}$ denotes the center of $s$ (there is only one $s$ now) the above solute has the following properties, as noted elsewhere. ${ }^{\text {a }}$

$$
\begin{gathered}
\rho_{s}{ }^{0}(\mathbf{r})=-\nabla_{r} \delta\left(\mathbf{r}-\mathbf{r}_{s}\right) \cdot \boldsymbol{u} \\
A_{s}\left(\mathbf{r}, \mathbf{r}^{\prime}\right)=\nabla_{r} \delta\left(\mathbf{r}-\mathbf{r}_{s}\right) \cdot \alpha \nabla_{r_{s}} \int d \mathbf{r}^{\prime} \delta\left(\mathbf{r}^{\prime}-\mathbf{r}_{s}\right) .
\end{gathered}
$$


The last term in (35) then becomes

$$
-\left\langle\nabla \Phi_{s_{1}-0}^{o p}\right\rangle_{1-0,0 p} \cdot\left(\alpha_{1}-\alpha_{0}\right)\left\langle\nabla \Phi_{s_{0}}\right\rangle_{0}
$$

For the above model we have ${ }^{12}$

$$
\begin{gathered}
F^{\gamma}=-\left[f /\left(1-f \alpha^{\gamma}\right)\right] \frac{1}{2}\left(\mathbf{u}^{2}\right), \\
f=\left(1 / a^{3}\right)[(2 D-2) /(2 D+1)], \\
-\left\langle\nabla \Phi_{s}\right\rangle=R=f \mathfrak{u} /(1-f \alpha),
\end{gathered}
$$

where $R$ is the well-known reaction field (of an equilibrium polarization system) acting on a solute dipole which is held fixed in position.

In state $[0], \boldsymbol{u}=\boldsymbol{u}_{0}$ and $D=D_{s}$, the static dielectric constant of the medium. In state $[1-0, o p], \boldsymbol{u}=\boldsymbol{u}_{1}-\boldsymbol{u}_{0}$ and $D=D_{\mathrm{op}}$, the square of the refractive index of the medium. We write $\alpha^{\gamma}=\alpha_{0}+\gamma\left(\alpha_{1}-\alpha_{0}\right)$.

Using (38) to (40) Eq. (35) becomes:

$$
\begin{array}{r}
\langle\Delta U\rangle_{0}=-\left(\frac{f_{\mathrm{op}}{ }^{2}}{\left(1-f_{\mathrm{op}} \alpha_{0}\right)^{2}} \frac{\boldsymbol{\mu}_{1-c^{2}}{ }^{2}}{2}+\frac{f_{s}{ }^{2}}{\left(1-f_{s} \alpha_{0}\right)^{2}} \frac{\boldsymbol{\mu}_{0}{ }^{2}}{2}\right. \\
\left.+\frac{f_{\mathrm{op}} \boldsymbol{\mu}_{1-\mathrm{c}}}{\left(1-f_{\mathrm{op}} \alpha_{0}\right)} \frac{f_{s} \boldsymbol{\mu}_{0}}{\left(1-f_{s} \alpha_{0}\right)}\right)\left(\alpha_{1}-\alpha_{0}\right),
\end{array}
$$

i.e., $\langle\Delta U\rangle_{0}=-\frac{1}{2}\left(\frac{f_{\mathrm{op}}}{1-f_{\mathrm{op}} \alpha_{0}} \boldsymbol{u}_{1-0}+\frac{f_{\mathrm{s}}}{1-f_{s} \alpha^{0}} \boldsymbol{u}_{0}\right)^{2}\left(\alpha_{1}-\alpha_{0}\right)$.

We note that when $\mu_{1-0}$ equals $-\mu_{0}$, i.e., when $\mu_{1}$ vanishes, and when $f_{8}$ equals $f_{\mathrm{op}}$, i.e., when the medium has no orientation polarization, (42) vanishes, as it should: When the medium and the final electronic state of the solute are both nonpolar, the polar term in the spectral shift should be independent of the polarizability of the final state of the solute $\alpha_{1}$. It is independent when (42) vanishes. Parenthetically, it may be noted that the difference of polarizabilities in the two electronic states still makes a contribution to the spectral shift in this instance, namely, via the nonpolar term in $U_{1}-U_{0}$ in Eq. (6). The contribution arises from differences in London dispersion forces between the solute in its two electronic states and the medium.

12 See C. J. F. Böttcher, Theory of Electrical Polarization (Elsevier Publishing Company, Inc., Amsterdam, 1952), p. 139.

\section{APPENDIX I. RELATION OF $\tau$ - TO TOTAL CONFIGURATIONAL CONTRIBUTION TO FREE ENERGY}

The total configurational contribution to the free energy of equilibrium systems is

$$
\begin{aligned}
-k T \ln \int \exp \left[\frac{-\left(U^{f}+U\right)}{k T}\right] d \tau d \tau^{f} \\
\quad=-k T \ln \int \exp \left[\frac{-\left(U^{f}+F_{c}\right)}{k T}\right] d \tau^{\prime},
\end{aligned}
$$

where $F_{c}$ is given by Eq. (3). [If any high-frequency coordinates are treated quantum mechanically, they should be excluded from $\tau^{f} ;(43)$ is then the value at any given value of these coordinates, there being a quantum mechanical distribution of the $\tau^{\prime}$. One then integrates over them in the appropriate quantum plus statistical mechanical fashion.]

In the case of nonequilibrium systems (with a similar qualification about any high frequency coordinates) the total configurational difference of nonequilibrium and equivalent equilibrium systems is:

$$
\frac{\int\left(U_{1}+U_{1}^{f}-U_{0}-U_{0}^{f}\right) \exp \left[\frac{-\left(U_{0}+U_{0}^{f}\right)}{k T}\right] d \tau d \tau^{f}}{\int \exp \left[\frac{-\left(U_{0}+U_{0}^{f}\right)}{k T}\right] d \tau d \tau^{f}}
$$

Since $U_{1}^{f}-U_{0}^{f}$ is independent of $\tau$, (44) becomes:

$$
\left\langle U_{1}^{f}-U_{0}^{f}\right\rangle_{0, f}+\left\langle F_{c}^{\text {non }}-F_{c_{0}}\right\rangle_{0, f}
$$

where \langle\rangle$_{0, f}$ denotes average with respect to

$$
\exp \left(\frac{-U_{0}^{f}}{k T}\right) / \int \exp \left(\frac{-U_{0}}{k T}\right) d \tau^{f}
$$

The polar contribution (7) to $F_{0}^{\text {non }}-F_{c_{0}}$ may be inserted into (45) or, when it is but weakly dependent on $\tau^{f}$, replaced by its value at the most probable value of $\tau^{f}$. In practice, the second of these procedures will usually be followed. The nonpolar contribution to $F_{c}{ }^{\text {non }}-F_{c},\left\langle U_{1}(0)-U_{0}(0)\right\rangle_{0, f}$ is also of much interest in certain problems and is to be added to the polar one. 